\title{
$S$-OPERATIONS IN REPRESENTATION THEORY $\left({ }^{1}\right)$
}

BY

\author{
EVELYN HUTTERER BOORMAN
}

ABSTRACT. For $G$ a group and $A^{G}$ the category of $G$-objects in a category $A$, a collection of functors, called " $S$-operations," is introduced under mild restrictions on $A$. With certain assumptions on $A$ and with $G$ the symmetric group $S_{k}$, one obtains a unigeneration theorem for the Grothendieck ring formed from the isomorphism classes of objects in $A^{S_{k}}$. For $A=$ finite-dimensional vector spaces over $C$, the result says that the representation ring $R\left(S_{k}\right)$ is generated, as a $\lambda$-ring, by the canonical $k$-dimensional permutation representation. When $A=$ finite sets, the $S$-operations are called " $\beta$-operations," and the result says that the Burnside ring $B\left(S_{k}\right)$ is generated by the canonical $S_{k}$-set if $\beta$-operations are allowed along with addition and multiplication.

A. Introduction. In the theory of linear representations of a finite group $G$, representations can be added, multiplied, and formed into a ring $R(G)$, the representation ring of $G$. In addition, $n$th symmetric power operations can be applied to any representation, and these operations can be extended to all elements of $R(G)$. Knutson [5] gives a detailed account of these operations in $R(G)$; Atiyah [1] discusses similar operations in the setting of vector bundles.

This paper attempts to generalize these notions. For any group $G$, a collection of operations on the category $\mathbf{A}^{\mathbf{G}}$ is defined under mild restrictions on A. In the case of linear representations of a finite group, these operations are combinations of symmetric powers, but, in general, they include other operations as well. Letting $G=S_{k}$ and with certain assumptions on $A^{S_{k}}$, one obtains the main result:

Corollary II.22. $\left\langle X_{k}\right\rangle=K_{0}\left(\mathrm{~A}^{\mathrm{S}_{\mathrm{k}}}\right)$.

Here, $K_{0}\left(A^{S_{k}}\right)$ is the Grothendieck ring formed from the isomorphism classes of objects in $\mathbf{A}^{\mathbf{S}_{\mathbf{k}}}, X_{k}$ is a particular object in $\mathbf{A}^{\mathbf{S}_{\mathbf{k}}}$, and $\left\langle X_{k}\right\rangle$ is the

Received by the editors November 11, 1973.

AMS (MOS) subject classifications (1970). Primary 20C30, $20 \mathrm{~J} 99$.

Key words and phrases. Representation ring, Grothendieck ring, $\lambda$-ring, Burnside ring.

(1) This research constitutes the author's doctoral thesis written at Columbia University under the direction of Professor Donald Knutson. The author wishes to thank Professor Knutson for his guidance and encouragement. 
subring of $K_{0}\left(\mathrm{~A}^{\mathrm{S}_{\mathrm{k}}}\right)$ obtained by applying the operations to $X_{k}$ and taking sums and products of the results. A principal application of this corollary is that $R\left(S_{k}\right)$ is generated by the canonical permutation representation $X_{k}$ if symmetric powers are included along with addition and multiplication. For the reader familiar with $\lambda$-rings, this statement says that $R\left(S_{k}\right)$ is generated by one element as a $\lambda$-ring [2], [5].

$\S \S I . B$ and I.C present some background on the two principal examples, the Burnside and representation rings of a finite group. Chapter II introduces the $S$-operations and explores their behavior; the main theorem and its Corollary II.22 are proved in $\S$ C. In Chapter III, Corollary II.22 is used to prove that $R\left(S_{k}\right)=$ $\left\langle X_{k}\right\rangle$ and $B\left(S_{k}\right)=\left\langle X_{k}\right\rangle$ for all $k \geqslant 1$. It is also shown that, in general, neither $B\left(S_{k}\right)$ nor $R\left(S_{k}\right)$ is unigenerated as a ring. Moreover, although $B\left(S_{k}\right)$ $=\left\langle X_{k}\right\rangle$, if one allows only symmetric power operations rather than all $S$-operations, one does not necessarily obtain all of $B\left(S_{k}\right)$.

B. The Burnside ring, $B(G)$. Let $G$ be a finite group. A $G$-set is a finite set $T$ together with a mapping $G \times T \rightarrow T$ such that $\left(g_{1} g_{2}\right) t=g_{1}\left(g_{2} t\right), 1 t$ $=t$, for all $g_{1}, g_{2} \in G, t \in T$. A morphism of $G$-sets, or $G$-map, is a set map $f: T \rightarrow T^{\prime}$, with $T$ and $T^{\prime} G$-sets, such that $f(g t)=g f(t)$ for all $g \in G$, $t \in T$. Two $G$-sets are said to be isomorphic if there is a $G$-map between them which is a set isomorphism. $G$-sets and $G$-maps clearly form a category.

EXAMPLES I.1. (i) Let $G$ be any finite group, $T$ any finite set. Then $T$ can be given the trivial action $g t=t$ for all $g \in G, t \in T$.

(i') In example (i), if $T$ has only one element, $T$ is denoted by $1_{G}$. (Of course, all one-element $G$-sets are isomorphic.)

(ii) Let $H$ be a subgroup of a finite group $G$. Then $G / H$, the set of left cosets of $H$ in $G$, is a $G$-set by the action $g(x H)=(g x) H$.

(iii) Let $S_{n}$ be the symmetric group on the symbols $1,2, \cdots, n$. Let $X_{n}$ be the set $\left\{x_{1}, x_{2}, \cdots, x_{n}\right\}$, and let $S_{n}$ act on $X_{n}$ by $\sigma x_{i}=x_{\sigma(i)}$. $X_{n}$ will be called the canonical $S_{n}$-set.

(iv) Let $G$ be any finite group. The empty set $\varnothing$ is clearly a $G$-set.

If $T_{1}$ and $T_{2}$ are $G$-sets, then the disjoint union $T_{1} \amalg T_{2}$ is a $G$-set, under the obvious action. On the other hand, every $G$-set can be decomposed into its G-orbits:

Proposition 1.2. Every $G$-set $T \neq \varnothing$ is of the form $\coprod_{i=1}^{n} T_{i}$, where $T_{i}$ is a transitive G-set. The $T_{i}$ 's are unique up to order. ( $A$ G-set $T$ is transitive if $T \neq \varnothing$ and if given $t_{1}, t_{2} \in T$ there is a.g $\in G$ such that $g t_{1}=$ $t_{2}$.)

Proposition 1.3. If $H$ is a subgroup of $G$, then $G / H$ is a transitive 
G-set. Conversely, every transitive $G$-set is of the form $G / H$ for some subgroup $H$ of $G$.

Proof. Given $g_{1} H, g_{2} H \in G / H,\left(g_{1} g_{2}^{-1}\right) g_{2} H=g_{1} H$. Hence $G / H$ is a transitive $G$-set.

Suppose $T$ is a transitive $G$-set. Let $t \in T$. Then $T=G t$. Let $G_{t}$ be the isotropy group of $t$, i.e., $G_{t}=\{g \in G \mid g t=t\}$. Then the map $T \rightarrow G / \dot{G}_{t}$ defined by $g t \mapsto g G_{t}$ is a $G$-isomorphism $\square$

Proposition I.4. $G / H \cong G / K$ as $G$-sets if and only if $H$ and $K$ are conjugate subgroups of $G$.

Proof. Suppose $H$ and $K$ are conjugate, i.e., $K=g_{1}^{-1} H g_{1}$ for some $g_{1} \in G$. Then the maps

$$
\begin{aligned}
\phi: G / H & \rightarrow G / K, \quad \psi: G / K & \rightarrow G / H, \\
g H & \mapsto\left(g g_{1}\right) K, \quad g K & \mapsto\left(g g_{1}^{-1}\right) H,
\end{aligned}
$$

are $G$-maps, and $\phi \circ \psi=1_{G / K}, \psi \circ \phi=1_{G / H}$. So $G / H \cong G / K$.

Conversely, assume $G / H \cong G / K$. Then there exist $G$-maps $\phi: G / H \rightarrow$ $G / K, \quad \psi: G / K \rightarrow G / H$ such that $\phi \circ \psi=1_{G / K}, \psi \circ \phi=1_{G / H}$. If $\phi(1 H)=$ $g_{1} K$, then $g_{1} K=h g_{1} K$ for all $h \in H$, so $g_{1}^{-1} H g_{1} \subset K$. Similarly $\phi(1 K)=$ $g_{2} H$ gives $g_{2}^{-1} K g_{2} \subset H$. Thus $g_{2}^{-1} g_{1}^{-1} H_{1} g_{2} \subset g_{2}^{-1} K g_{2} \subset H$. Since $g_{2}^{-1} g_{1}^{-1} H g_{1} g_{2}$ has the same number of elements as $H, g_{2}^{-1} g_{1}^{-1} \mathrm{Hg}_{1} g_{2}=g_{2}^{-1} \mathrm{Kg}_{2}=H$.

If $T_{1}$ and $T_{2}$ are $G$-sets, then the cartesian product $T_{1} \times T_{2}$ is a $G$-set under the obvious action. The Burnside ring of $G, B(G)$, consists of all finite formal sums, $\Sigma_{i} n_{i}\left[T_{i}\right] \quad\left(n_{i} \in Z\right)$, of $G$-sets $T_{i}$, modulo the relations

(i) $\left[T_{1}\right]=\left[T_{2}\right]$ if $T_{1} \cong T_{2}$ as $G$-sets,

(ii) $\left[T_{1} \amalg T_{2}\right]=\left[T_{1}\right]+\left[T_{2}\right]$.

$B(G)$ is clearly an abelian group; the cartesian product, together with $\mathbf{1}_{G}$, gives $B(G)$ the structure of a commutative ring with identity, i.e., $\left[T_{1}\right]\left[T_{2}\right]=$ $\left[T_{1} \times T_{2}\right]$. Whenever no confusion could arise, the brackets will be omitted.

Propositions I.2, I.3, and I.4 imply

Proposition I.5. Let $\left\{H_{\alpha}\right\}$ be a set of representatives of the conjugacy classes of subgroups of $G$. Then $B(G)$ is a free $Z$-module with basis $\left\{\left[G / H_{\alpha}\right]\right\}$.

The rest of this section is devoted to defining a set map $h_{n}: B(G) \rightarrow B(G)$ for each integer $n \geqslant 0$. For any $G$-set $T$, the set $T_{G}$ is defined to be the collection of elements of $T$ with the identification $t_{1} \sim t_{2}$ iff $G t_{1}=G t_{2}$.

Let $T$ be a $G$-set. Then $T^{n}=T \times T \times \cdots \times T$ ( $n$ times) is a $G$-set and also an $S_{n}$-set via $\sigma\left(t_{1}, \cdots, t_{n}\right)=\left(t_{\sigma-1(1)}, \cdots, t_{\sigma-1(n)}\right)$ for $\sigma \in S_{n}$. For each integer $n \geqslant 1$, let $h_{n}(T)$ denote $\left(T^{n}\right)_{S_{n}} ; h_{n}(T)$ is thus the $n$th 
symmetric power of $T$. Since the $G$ - and $S_{n}$ - actions on $T^{n}$ commute, $h_{n}(T)=\left(T^{n}\right)_{S_{n}}$ is actually a $G$-set. Clearly $h_{n}$ sends isomorphic $G$-sets to isomorphic $G$-sets. Finally, define $h_{0}(T)$ to be $1_{G}$ for all $G$-sets $T$.

If $T_{1}, T_{2}$ are $G$-sets, then

$$
h_{n}\left(T_{1} \amalg T_{2}\right)=\coprod_{i=0}^{n}\left(h_{i}\left(T_{1}\right) \times h_{n-i}\left(T_{2}\right)\right) .
$$

$h_{n}$ can now be defined on any element of $B(G)$ by the following construction:

Define

$$
H_{n}:(G \text {-sets }) \times(G \text {-sets }) \rightarrow B(G)
$$

inductively by

$$
\begin{aligned}
& H_{0}\left(T_{1}, T_{2}\right)=1_{G}, \\
& H_{n}\left(T_{1}, T_{2}\right)=h_{n}\left(T_{1}\right)-\sum_{i=1}^{n-1} H_{i}\left(T_{1}, T_{2}\right) h_{n-i}\left(T_{2}\right) \text { for } n>0 .
\end{aligned}
$$

Clearly,

$$
T_{1} \cong U_{1}, T_{2} \cong U_{2} \Rightarrow H_{n}\left(T_{1}, T_{2}\right)=H_{n}\left(U_{1}, U_{2}\right) \text { for all } n \geqslant 0 .
$$

In addition, an induction argument and the "addition formula" above give

$$
H_{n}\left(T_{1} \amalg T, T_{2} \amalg T\right)=H_{n}\left(T_{1}, T_{2}\right), \quad H_{n}(T, \varnothing)=h_{n}(T)
$$

for all $n \geqslant 0$ and $G$-sets $T_{1}, T_{2}, T$.

An arbitrary element of $B(G)$ looks like $T_{1}-T_{2}$, where $T_{1}$ and $T_{2}$ are $G$-sets. If $T_{1}-T_{2}=U_{1}-U_{2}$, then $T_{1} \amalg U_{2} \cong U_{1} \amalg T_{2}$, so

$$
\begin{aligned}
H_{n}\left(T_{1}, T_{2}\right) & =H_{n}\left(T_{1} \amalg U_{2}, T_{2} \amalg U_{2}\right) \\
& =H_{n}\left(U_{1} \amalg T_{2}, T_{2} \amalg U_{2}\right)=H_{n}\left(U_{1}, U_{2}\right) .
\end{aligned}
$$

Thus $H_{n}\left(T_{1}, T_{2}\right)$ depends only on $T_{1}-T_{2}$. Therefore, define $h_{n}\left(T_{1}-T_{2}\right)=$ $H_{n}\left(T_{1}, T_{2}\right)$. Then $h_{n}: B(G) \rightarrow B(G)$ is a well-defined set map and coincides with its former definition if $T \in B(G)$ is actually a $G$-set.

C. The representation ring, $R(G)$. Let $G$ be a finite group. A (linear) representation of $G$ (over $C$ ) is a finite-dimensional vector space $V$ over $C$, together with a group homomorphism $\rho: G \rightarrow$ Aut $V . V$ is called a $G$-module, and $\rho$ gives an action of $G$ on $V$. One usually writes

$$
V \stackrel{g}{\rightarrow} V, \quad v \mapsto g v
$$

instead of

$$
V \stackrel{\rho(g)}{\longrightarrow} V, \quad v \mapsto \rho(g) v
$$


A $G$-module map is a linear transformation $f: V \rightarrow V^{\prime}$, with $V$ and $V^{\prime} G$-modules, such that $f(g v)=g f(v)$ for all $g \in G, v \in V$. Two $G$-modules are said to be isomorphic if there exists a $G$-module map between them which is also a vector space isomorphism. $G$-modules and $G$-module maps clearly form a category.

EXAMPLES I.6. (i) Let $G$ be a finite group, $V$ a finite-dimensional vector space. $V$ can be given the trivial action $g v=v$ for all $g \in G, v \in V$.

(i') A special case of example (i) is $V=0$.

(i") In example (i), if $\operatorname{dim} V=1, V$ is denoted by $1_{G}$.

(ii) Let $G=S_{n}$. Let $V$ have basis $\left\{v_{1}, \cdots, v_{n}\right\}$, and let $S_{n}$ act by $\sigma v_{i}=v_{\sigma(i)}$ for $\sigma \in S_{n}$. This representation $V$ will be called the canonical $S_{n}$-module, and denoted $X_{n}$.

(ii') More generally, suppose $\rho: G \rightarrow S_{n}$ is a group homomorphism. ( $\rho$ is called a permutation representation.) By composing this homomorphism with the one in example (ii), one obtains a linear representation of $G, G \stackrel{\rho}{\longrightarrow} S_{n} \rightarrow$ Aut $X_{n}$. Since a $G$-set $T$ consisting of $n$ elements is a group homomorphism $G \rightarrow S_{n}$, the concept of $G$-set is the same as the concept of permutation representation of $G$.

A $G$-module $V$ is reducible if $V=0$ or if there is a subspace $W$ of $V$ such that $G W \subset W$, with $W \neq 0$ and $W \neq V$. If $V$ is not reducible, it is called irreducible.

If $V_{1}, V_{2}$ are $G$-modules, then the vector space coproduct $V_{1} \amalg V_{2}$ is a $G$-module via the obvious action. A $G$-module $V$ is said to be decomposable if $V \cong V_{1} \amalg V_{2}$ as a $G$-module, where $V_{i} \neq 0$. Propositions I.7-I.9 can be found in any book on group representation theory (see [5], [8]).

Proposition I.7 (MASCHKE). If $V \neq 0$ is reducible, then $V$ is decomposable.

Proposition I.8. Every G-module $V \neq 0$ can be expressed as a finite coproduct $V=\coprod_{i=1}^{n} V_{i}$, where each $V_{i}$ is an irreducible G-module. The $V_{i}$ 's are unique (up to order).

Proposition 1.9. The number of irreducible representations of $G$ is equal to the number of conjugacy classes of $G$.

For $G$-modules $V_{1}, V_{2}, V_{1} \otimes V_{2}$ is a $G$-module via $g\left(v_{1} \otimes v_{2}\right)=g v_{1} \otimes$ $g v_{2}$. The representation ring of $G, R(G)$, consists of all finite formal sums $\Sigma_{i} n_{i}\left[V_{i}\right]\left(n_{i} \in Z\right)$, of $G$-modules $V_{i}$, modulo the relations

(i) $\left[V_{1}\right]=\left[V_{2}\right]$ if $V_{1} \cong V_{2}$ as $G$-modules,

(ii) $\left[V_{1} \amalg V_{2}\right]=\left[V_{1}\right]+\left[V_{2}\right]$.

$R(G)$ is clearly an abelian group; the tensor product, together with $1_{G}$, gives 
$R(G)$ the structure of a commutative ring with identity, that is $\left[V_{1}\right]\left[V_{2}\right]=$ $\left[V_{1} \otimes V_{2}\right]$. The brackets will usually be omitted.

Propositions 1.8 and 1.9 imply

Proposition I.10. Let Irrep $G=$ the set of isomorphism classes of irreducible G-modules. Then $R(G)$ is a free $Z$-module with basis $\{[V] \mid V \in$ Irrep $G$ \}. The rank of $R(G)=$ the number of conjugacy classes of $G$.

As in the case of $B(G)$, symmetric power operations $h_{n}: R(G) \rightarrow R(G)$ can be introduced. For any $G$-module $V$, define the vector space $V_{G}$ to be $V / W$, where $W$ is the subspace of $V$ generated by $\{v-g v \mid v \in V, g \in G\}$. The vector spaces $V_{G}$ and $V^{G}$, where $V^{G}$ is the subspace of $V$ fixed by $G$, are seen to be isomorphic by the fact that the linear transformation $Y$ : $V \rightarrow V$ defined by

$$
Y(v)=\frac{1}{|G|} \sum_{g \in G} g V
$$

has image $V^{G}$ and kernel $W$. In the case of sets, however, the corresponding objects $T_{G}$ and $T^{G}$ are not generally isomorphic.

For any $G$-module $V, V^{\otimes n}=V \otimes \cdots \otimes V$ ( $n$ times) is a $G$-module and also an $S_{n}$-module via $\sigma\left(v_{1} \otimes \cdots \otimes v_{n}\right)=v_{\sigma^{-1}(1)} \otimes \cdots \otimes v_{\sigma^{-1}(n)}$ for $\sigma \in S_{n}$. For each positive integer $n$, let $h_{n}(V)$ denote $\left(V^{\otimes n}\right)_{S_{n}} ; h_{n}(V)$ is thus the $n$th symmetric power of $V$. Since the $G$ - and $S_{n}$ - actions on $V^{\otimes n}$ commute, $h_{n}(V)=\left(V^{\otimes n}\right)_{S_{n}}$ is a $G$-module. Define $h_{0}(V)$ to be $1_{G}$ for all $G$-modules $V$. Clearly $h_{n}$ sends isomorphic $G$-modules to isomorphic $G$ modules.

For $G$-modules $V_{1}$ and $V_{2}$,

$$
h_{n}\left(V_{1} \amalg V_{2}\right)=\prod_{i=0}^{n}\left(h_{i}\left(V_{1}\right) \otimes h_{n-i}\left(V_{2}\right)\right) .
$$

As in the $G$-set case, $h_{n}$ can be defined on any element $V_{1}-V_{2}$ of $R(G)$ by defining $H_{n}$ : ( $G$-modules) $\times(G$-modules) $\rightarrow R(G)$ inductively by

$$
\begin{aligned}
& H_{0}\left(V_{1}, V_{2}\right)=1_{G}, \\
& H_{n}\left(V_{1}, V_{2}\right)=h_{n}\left(V_{1}\right)-\sum_{i=0}^{n-1} H_{i}\left(V_{1}, V_{2}\right) h_{n-i}\left(V_{2}\right) \text { for } n>0,
\end{aligned}
$$

and then using the "addition formula" above to show that $H_{n}\left(V_{1}, V_{2}\right)$ depends only on $V_{1}-V_{2}$.

$G$-sets and G-modules are examples of the category discussed in Chapter II. There, a family of functors, called $S$-operations, is defined. In the case of 
$G$-modules, these $S$-operations turn out to be sums and products of symmetric powers $h_{n}$. In fact, by applying these operations to the canonical $S_{k}$-module $X_{k}$, one can obtain every element in $R\left(S_{k}\right)$ (see III, $\S A$ ).

In the case of $G$-sets, however, the $S$-operations include more than symmetric powers. In III, $\S \mathrm{B}$, one sees that applying sums and products of symmetric power operations $h_{n}$ to the canonical $S_{k}$-set $X_{k}$ does not always give all of $B\left(S_{k}\right)$, whereas applying all the $S$-operations to $X_{k}$ does.

\section{S-OPERATIONS}

A. The category $\mathbf{A}^{\mathbf{G}}$ and functors $\phi_{W_{n}}: \mathbf{A}^{\mathbf{G}} \rightarrow \mathbf{A}^{\mathbf{G}}$. Let $G$ be a group, and $\mathbf{A}$ a category. $\mathbf{A} G$-object in $\mathbf{A}$ is an object $A$ in $\mathbf{A}$, together with morphisms $A \stackrel{\rho_{g}}{\longrightarrow} A$ for all $g \in G$, satisfying $\rho_{g h}=\rho_{g} \circ \rho_{h}, \rho_{1}=1_{A}$. $A \stackrel{\rho_{g}}{\longrightarrow} A$ is usually written $A \stackrel{g}{\longrightarrow} A$.

A $G$-map, or $G$-morphism, is a morphism $f: A \rightarrow B$ in A, with $A$ and $B G$-objects, such that $f g=g f$ for all $g \in G$. The category of $G$-objects and $G$-maps in $A$ is denoted $A^{\mathbf{G}}$.

The aim of this section is to define a collection of functors from $A^{\mathbf{G}}$ to $\mathbf{A}^{\mathbf{G}}$, under certain assumptions on $\mathbf{A}$. The reader is referred to [6] for a reference on category theory.

Recall that given two morphisms $\alpha, \beta: A \rightarrow B, \mu: B \rightarrow K$ is a coequalizer for $\alpha$ and $\beta$ if $\mu \alpha=\mu \beta$, and if whenever $\mu^{\prime}: B \rightarrow K^{\prime}$ satisfies $\mu^{\prime} \alpha=$ $\mu^{\prime} \beta$, then there is a unique morphism $\gamma: K \rightarrow K^{\prime}$ such that $\gamma \mu=\mu^{\prime}$. Given two morphisms $f_{1}: A \rightarrow B_{1}, f_{2}: A \rightarrow B_{2}$, a commutative diagram

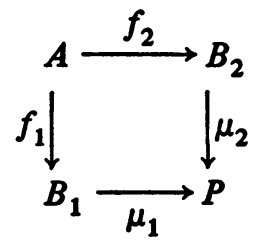

is called a pushout for $f_{1}$ and $f_{2}$ if for every commutative diagram

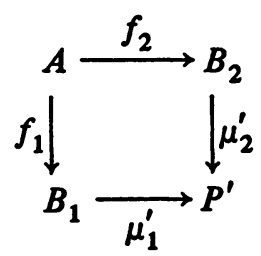

there is a unique morphism $\gamma: P \rightarrow P^{\prime}$ such that $\mu_{1}^{\prime}=\gamma u_{1}$ and $\mu_{2}^{\prime}=\gamma u_{2}$.

LEMMA II.1. Let A be a category with coequalizers and finite coproducts. Then

A has pushouts. 
Proof. Consider

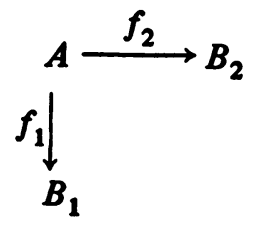

The coproduct $B_{1} \amalg B_{2}$, together with the canonical morphisms $i_{j}: B_{j} \rightarrow$ $B_{1} \amalg B_{2}, j=1,2$, gives morphisms $i_{j} \circ f_{j}: A \rightarrow B_{1} \amalg B_{2}, j=1,2$. Let $\mu: B_{1} \amalg B_{2} \rightarrow K$ be the coequalizer for $i_{1} \circ f_{1}$ and $i_{2} \circ f_{2}$. Then $\mu \circ$ $\left(i_{1} \circ f_{1}\right)=\mu \circ\left(i_{2} \circ f_{2}\right)$ gives a commutative diagram

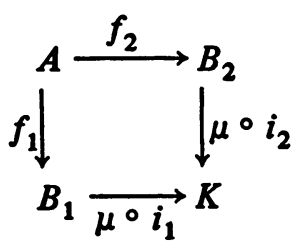

The fäct that this diagram is actually a pushout follows from the definitions of coproduct and coequalizer.

Given a family $\left\{\mu_{i}: A \rightarrow A_{i}\right\}_{i \in I}$ of epimorphisms, $\mu: A \rightarrow A^{\prime}$ is the cointersection of the family if for each $i \in I$ there exist morphisms $\nu_{i}: A_{i} \rightarrow$ $A^{\prime}$ such that $\mu=\nu_{i} \mu_{i}$, and if every morphism $A \rightarrow B$ which factors through each $\mu_{i}$ factors uniquely through $\mu$.

LEMmA II.2. If A has pushouts, then A has finite cointersections.

Proof. It suffices to show existence for a family of two epimorphisms $\mu_{1}: A \rightarrow A_{1}, \mu_{2}: A \rightarrow A_{2}$. Let

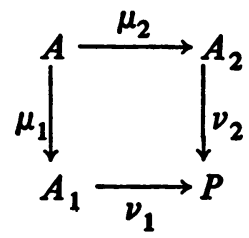

be the pushout for $\mu_{1}$ and $\mu_{2}$. Then $v_{1} \mu_{1}=\nu_{2} \mu_{2}: A \rightarrow P$ is the cointersection of $\mu_{1}$ and $\mu_{2}$ by the definition of pushout.

Let $F_{G}: A \rightarrow A^{G}$ be the functor which sends $A \in \mathrm{A}$ to $A \in \mathrm{A}^{\mathrm{G}}$ by letting $A \stackrel{g}{\longrightarrow} A$ be $A \stackrel{1 A}{\longrightarrow} A$ for all $g \in G$. Let $V \in A^{\mathbf{G}}$. A $G$-orbit space of $V$ is a pair $(O, \pi)$, where $O \in A$ and $\pi \in \operatorname{Mor}_{A} G\left(V, F_{G}(O)\right)$, such that whenever $X \in A$ and $f \in \operatorname{Mor}_{A} G\left(V, F_{G}(X)\right)$ there is a unique $\phi \in$ $\operatorname{Mor}_{A}(O, X)$ such that $F_{G}(\phi) \circ \pi=f$. When such an $O$ exists, it is of course unique up to natural isomorphism and is denoted $V_{G}$. 
Proposition II.3. Let $G$ be a finite group and let $\mathbf{A}$ have coequalizers and finite coproducts. Then $\left(V_{G}, \pi\right)$ exists for all $V \in \mathbf{A}^{\mathbf{G}}$.

Proof. For each pair of distinct elements $g_{i}, g_{j} \in G$, let $\mu_{g_{i}, g_{j}}: V \rightarrow$ $K_{g_{i}, g_{j}}$ be a coequalizer for the morphisms $g_{i}, g_{j}: V \rightarrow V$. Each $\mu_{g_{i}, g_{j}}$ is an epimorphism since every coequalizer is. Let $\pi: V \rightarrow O$ be the cointersection (exists by Lemmas II.1, II.2) of the finite family $\left\{\mu_{g_{i}, g_{j}}: V \rightarrow K_{g_{i}, g_{j}}\right\}_{g_{i}} \neq g_{j}$ in A. This construction gives $\left(V_{G}, \pi\right)$ :

For each $g \in G, \mu_{g, 1} g=\mu_{g, 1} 1_{V}=\mu_{g, 1}$, so $\pi g=\pi$ for all $g \in G$. Hence $\pi \in \operatorname{Mor}_{A} G\left(V, F_{G}(O)\right)$. If $f \in \operatorname{Mor}_{A} G\left(V, F_{G}(X)\right)$, then $f g_{i}=f g_{j}$ for all $g_{i}$, $g_{j} \in G$, so $f$ factors through each $\mu_{g_{i}, g_{j}}$. Thus there is a unique $\phi \in$ $\operatorname{Mor}_{A}(O, X)$ such that $\phi^{\circ} \pi=f$. $\square$

RemarK II.4. Proposition II.3 says there is a functor ()$_{G}: \mathbf{A}^{\mathbf{G}} \rightarrow \mathbf{A}$ left adjoint to $F_{G}: \mathbf{A} \rightarrow \mathbf{A}^{\mathbf{G}}$, i.e., $\operatorname{Mor}_{\mathbf{A}} \mathbf{G}\left(V, F_{G}(X)\right) \approx \operatorname{Mor}_{\mathbf{A}}\left(V_{G}, X\right)$, natural in arguments $V$ and $X$.

For each integer $n \geqslant 1$ and each $W_{n} \in \mathrm{A}^{\mathrm{S}_{\mathrm{n}}}$ ( $S_{n}$ is the symmetric group), a functor $\phi_{W_{n}}: \mathbf{A}^{\mathbf{G}} \rightarrow \mathbf{A}^{\mathbf{G}}$ will be defined. To do so, assume that $\mathbf{A}$ has not only coequalizers and finite coproducts but, also a "tensor product" 1 , that is, a functor $1: \mathbf{A} \times \mathbf{A} \rightarrow \mathbf{A}$ which is coherently associative and commutative (see [7, Chapter I]), and which distributes with the coproduct. This insures natural isomorphisms

$$
\begin{aligned}
\left(A_{1} \perp A_{2}\right) \perp A_{3} & \approx A_{1} \perp\left(A_{2} \perp A_{3}\right), \\
A_{1} \perp A_{2} & \approx A_{2} \perp A_{1}, \\
A_{1} \perp\left(A_{2} \amalg A_{3}\right) & \approx\left(A_{1} \perp A_{2}\right) \amalg\left(A_{1} \perp A_{3}\right),
\end{aligned}
$$

such that isomorphisms between products of several factors, obtained by successively applying the above, are the same.

Fix $W_{n} \in \mathrm{A}^{\mathrm{S}_{\mathrm{n}}}$. Let $T \in \mathrm{A}$ and let $T^{\perp n}=T \perp T \perp \cdots \perp T$ ( $n$ times). $T^{\perp n} \in A^{S_{n}}$ via the natural isomorphisms which permute its factors. Since $\perp$ is a functor $\mathbf{A} \times \mathbf{A} \rightarrow \mathbf{A}$ it induces a functor $1: \mathbf{A}^{\mathbf{G}} \times \mathbf{A}^{\mathbf{G}} \rightarrow \mathbf{A}^{\mathbf{G}}$ for any group $G$; that is if $A \stackrel{g}{\longrightarrow} A, B \stackrel{g}{\longrightarrow} B$, then $A \perp B \stackrel{g \perp g}{\longrightarrow} A \perp B$ gives $A \perp B$ a well-defined $G$-action by the functoriality of $\perp$. Hence $W_{n} \perp T^{\perp n} \in$ $\mathrm{A}^{\mathrm{S}_{\mathrm{n}}}$. Defining $\phi_{W_{n}}(T)$ to be $\left(W_{n} \perp T^{\perp n}\right)_{S_{n}}$, one obtains a functor $\phi_{W_{n}}$ : A $\rightarrow$ A. (For $f: T \rightarrow T^{\prime}, \phi_{W_{n}}(f):\left(W_{n} \perp T^{n}\right)_{S_{n}} \rightarrow\left(W_{n} \perp T^{\prime n}\right)_{S_{n}}$ is the obvious map.)

If $T \in \mathrm{A}^{\mathrm{G}}, T$ comes with morphisms $T \stackrel{g}{\longrightarrow} T$ for all $g \in G$, which induce morphisms

$$
\phi_{W_{n}}(T) \stackrel{\phi_{W_{n}}(g)}{\longrightarrow} \phi_{W_{n}}(T)
$$


for all $g \in G$. Since $\phi_{W_{n}}$ is a functor, the maps $\phi_{W_{n}}(g)$ define a $G$-action on $\phi_{W_{n}}(T)$. Thus one has a functor $\phi_{W_{n}}: \mathbf{A}^{\mathbf{G}} \rightarrow \mathbf{A}^{\mathbf{G}}$ for any group $G$.

' In conclusion, then, if $G$ is any group and if $A$ has coequalizers, finite coproducts, and a "tensor product" 1 , then for each positive integer and each $W_{n} \in \mathrm{A}^{\mathrm{S}_{\mathbf{n}}}$, one has a functor $\phi_{W_{n}}: \mathbf{A}^{\mathbf{G}} \rightarrow \mathrm{A}^{\mathbf{G}}$ defined by $\phi_{W_{n}}(T)=$ $\left(W_{n} \perp T^{\perp n}\right)_{S_{n}}$

B. The behavior of the functors $\phi_{W_{n}}$. The purpose of this section is to investigate the behavior of the functors $\phi_{W_{n}}$. To do so, one first introduces induced objects.

Suppose $H \subset G$ are groups. $A \in \mathbf{A}^{\mathbf{G}}$ may be viewed as an $H$-object via the inclusion $H \hookrightarrow G$, giving rise to a functor $\operatorname{Res}_{H}^{G}: \mathbf{A}^{\mathbf{G}} \rightarrow \mathbf{A}^{\mathbf{H}}$. Let $W \in$ $\mathbf{A}^{\mathbf{H}}$. An induced object of $W$ is a pair $(V, \psi)$, where $V \in \mathbf{A}^{\mathbf{G}}$ and $\psi \in$ $\operatorname{Mor}_{\mathrm{A}^{\mathrm{H}}}\left(W, \operatorname{Res}_{\mathrm{H}}^{\mathrm{G}} V\right)$ such that whenever $X \in \mathrm{A}^{\mathbf{G}}$ and $f \in \operatorname{Mor}_{\mathrm{A}^{\mathrm{H}}}\left(W, \operatorname{Res}_{\mathrm{H}}^{G} X\right)$ there is a unique $\phi \in \operatorname{Mor}_{A} G(V, X)$ satisfying $\left(\operatorname{Res}_{H}^{G} \phi\right) \circ \psi=f$. When such a $V$ exists, it is unique up to natural isomorphism and is denoted $\operatorname{Ind}_{\mathbf{H}}^{G} W$.

Proposition II.5. Let $H \subset G$ be finite groups and let $\mathbf{A}$ have coequalizers and finite coproducts. Then $\left(\operatorname{Ind}_{\mathbf{H}}^{\mathbf{G}} W, \psi\right)$ exists for all $W \in \mathbf{A}^{\mathbf{H}}$.

Proof. Let $W \in \mathbf{A}^{\mathbf{H}}$. Form the coproduct of $W$ with itself $|G|$ times to obtain the object $\amalg_{x \in G} W_{x}$ in $\mathbf{A}$, which is in $\mathbf{A}^{\mathbf{G}}$ via the maps $\amalg W_{x} \stackrel{{ }_{g}}{\longrightarrow}$ $\amalg W_{x}$, for all $g \in G$, which permute the factors; more precisely, ${ }^{*} g$ is induced by the maps ${ }^{*} g_{x}: W_{x} \rightarrow W_{g x} \hookrightarrow \amalg_{x \in G} W_{x}$, where the first morphism is $1_{W}$ and the second is the canonical map associated with the coproduct. In the future, $W_{x} \stackrel{1^{1}}{\longrightarrow} W_{y}$ will be denoted $1_{x}^{y}$. For $h \in H$, let $\amalg W \stackrel{h^{*}}{\longrightarrow} \amalg W_{x}$ be the map induced from maps

$$
h_{x}^{*}: w_{x} \stackrel{1_{x}^{x h}}{\longrightarrow} w_{x h} \hookrightarrow \amalg w_{x},
$$

and let $\amalg W_{x} \stackrel{h}{\longrightarrow} \amalg W_{x}$ be induced from the maps $h_{x}: W_{x} \stackrel{h}{\longrightarrow} W_{x} \hookrightarrow \amalg W_{x}$, where the first map is just the action of $H$ on $W$.

Observe that $h^{* *} g={ }^{*} g h^{*}$ and $h\left({ }^{*} g\right)={ }^{*} g h$ for all $g \in G, h \in H$. For $h \in H$, let $\mu_{h}: \amalg W_{x} \rightarrow K_{h}$ be the coequalizer of $h^{*}$ and $h$. Since ${ }_{\theta}\left(\mu_{h}^{*} g\right) h^{*}$ $=\mu_{h} h^{* *} g=\left(\mu_{h} h\right)^{*} g=\left(\mu_{h}^{*} g\right) h$ for $g \in G$, there is a unique map $K_{h} \stackrel{g}{\longrightarrow} K_{h}$ such that the triangle

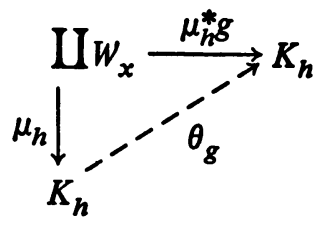

commutes. One thus obtains a map $\theta_{g}$ for each $g \in G$. The uniqueness of each 
$\theta_{g}$ implies $\theta_{1}=1_{K_{h}}$ and $\theta_{g_{1} g_{2}}=\theta_{g_{1}} \theta_{g_{2}}$. Hence $K_{h} \in A^{G}$ and $\mu_{h}$ is a G-map.

Let $\mu: \quad \amalg W_{x} \rightarrow K$ be the cointersection (exists by Lemmas II.1, II.2) of the finite family of epimorphisms $\left\{\mu_{h}\right\}_{h \in H}$ in A. Since $\mu$ factors through each $\mu_{h}, \mu^{*} g$ does also; hence for each $g \in G$, there is a unique map $K \stackrel{\rho_{g}}{\longrightarrow}$ $K$ such that the triangle

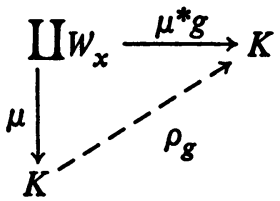

commutes. The uniqueness of each $\rho_{g}$ makes $K$ a $G$-object and therefore $\mu$ a $G$-map.

Let $\psi: W \rightarrow \operatorname{Res}_{H}^{G} K$ be the map $\mu_{1}: W_{1} \rightarrow K$ which comes from $\mu: \amalg_{x \in G} W_{x} \rightarrow K$. (Here and elsewhere, $W$ is identified with $W_{1}$.) $K=$ $\operatorname{Ind}_{H}^{G} W$ by the following argument:

To show that $\psi$ is an $H$-map, one must show that $\rho_{h} \psi=\psi h$. The last commutative triangle and the definition of $\mu$ give $\rho_{h} \psi=\rho_{h} \mu_{1}=\mu^{*} h_{1}=\mu h_{1}=$ $\mu_{1} h=\psi h$.

Suppose $f: W \rightarrow \operatorname{Res}_{H}^{G} X$ is an $H$-map. One can show that there is a unique $G$-map $\tau: \quad \amalg W_{x} \rightarrow X$ such that the triangle

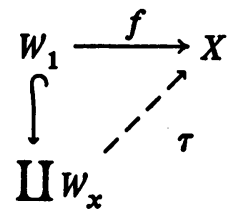

commutes, as follows:

If such a $\tau$ exists, then for each $x \in G$, the square

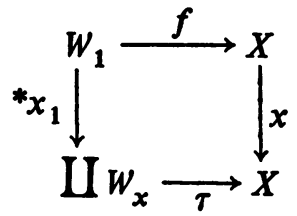

commutes. Hence $\tau_{x}=x f 1_{x}^{1}$ for all $x \in G$. Thus $\tau$ is unique. For existence, define $\tau$ by $\tau_{x}=x f 1_{x}^{1}$ for all $x \in G$.

Moreover, $\tau h^{*}=\tau h$ for all $h \in H$ since $\left(\tau h^{*}\right)_{x}=\tau_{x h} 1_{x}^{x h}=x h f 1_{x h}^{1} 1_{x}^{x h}$ $=x h f 1_{x}^{1},(\tau h)_{x}=\tau_{x} h=x f 1_{x}^{1} h=x f h 1_{x}^{1}$, and $f$ is an $H$-map. Hence $\tau$ factors through $\mu_{h}$ for all $h \in H$. It follows that there is a unique $\phi: K \rightarrow X$ satisfying $\tau=\phi \mu$. By the fact that $\mu$ and $\tau$ are $G$-maps and by the uniqueness of $\phi, \phi$ is itself a $G$-map: $\left(g \phi \rho_{g^{-1}}\right) \mu=g \phi \mu^{*} g^{-1}=g \tau g^{-1}=g g^{-1} \tau=\tau \Rightarrow g \phi \rho_{g-1}=$ $\phi$, or, $g \phi=\phi \rho_{g}$. 
The commutativity of the two small triangles in the figure

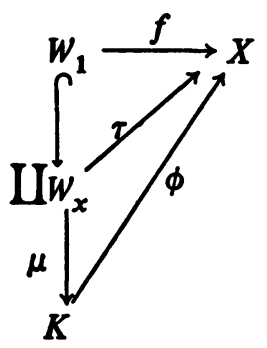

implies the commutativity of the large triangle. Hence $\phi$ is a $G$-map satisfying $\left(\operatorname{Res}_{H}^{G} \phi\right) \circ \psi=f$. In addition, if $\phi_{1}$ makes the diagram

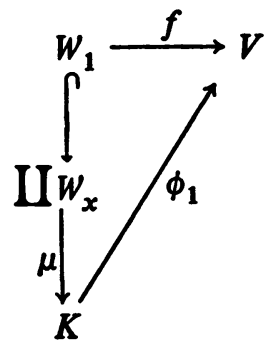

commute, then $\phi_{1} \mu=\tau$ by uniqueness of $\tau$, and so $\phi_{1}=\phi$ by uniqueness of $\varnothing . \square$

Remark II.6. Proposition II.5 says there is a functor $\operatorname{Ind}_{H}^{G}: \mathbf{A}^{\mathbf{H}} \rightarrow \mathbf{A}^{\mathbf{G}}$ left adjoint to $\operatorname{Res}_{H}^{G}: \mathbf{A}^{\mathbf{G}} \rightarrow \mathbf{A}^{\mathbf{H}}$, i.e., $\operatorname{Mor}_{\mathbf{A}^{\mathbf{H}}}\left(W, \operatorname{Res}_{H}^{G} V\right) \approx \operatorname{Mor}_{\mathbf{A}^{G}}{ }\left(\operatorname{Ind}_{H}^{G} W, V\right)$, natural in arguments $W$ and $V$.

Proposition II.7. Let $G$ be a group and $\mathbf{A}$ a category with finite coproducts. Suppose $V \in \mathrm{A}^{\mathrm{G}}$ and $V=\amalg_{i=1}^{n} W_{i}$ as an object in $\mathrm{A}$. Assume $G$ permutes the $W_{i}$ 's transitively, that is, each $g_{i}: W_{i} \rightarrow \amalg_{i=1}^{n} W_{i}$ looks like $W_{i}$ $\rightarrow W_{j} \hookrightarrow \amalg_{i=1}^{n} W_{i}$ for some $j$ and some morphism $W_{i} \rightarrow W_{j}$, and given any $i, j$, there is a $g \in G$ such that $g_{i}: W_{i} \rightarrow W_{j} \hookrightarrow \amalg_{i=1}^{n} W_{i}$.

Let $W_{i_{0}}$ be one of the $W_{i}$ 's and let $H$ be its isotropy group, i.e., $H=$ $\left\{g \in G \mid g_{i_{0}}: W_{i_{0}} \rightarrow W_{i_{0}} \hookrightarrow \amalg W_{i}\right\}$. Then as an object in $\mathbf{A}^{\mathbf{G}}, V=\operatorname{Ind}_{H}^{G} W_{i_{0}}$.

Proof. If $g_{i}: W_{i} \rightarrow W_{j} \hookrightarrow \amalg W_{i}$, denote the map $W_{i} \rightarrow W_{j}$ by $g_{i}^{j}$. Since $g g^{-1}=g^{-1} g=1_{v}, g_{i}: W_{i} \rightarrow W_{j} \hookrightarrow \amalg W_{t}$ implies $g_{j}^{-1}: W_{j} \rightarrow W_{i} \hookrightarrow$ $\amalg W_{i}$, and $g_{i}^{j}\left(g^{-1}\right)_{j}^{i}=1_{W_{j}},\left(g^{-1}\right)_{j i}^{i} g_{i}^{j}=1_{W_{i}}$

Let $\psi: W_{i_{0}} \rightarrow \operatorname{Res}_{H}^{G} V$ be the canonical map $W_{i_{0}} \hookrightarrow \amalg_{i=1}^{n} W_{i} \cdot \psi$ is clearly an $H$-map. To show $V=\operatorname{Ind}_{H}^{G} W_{i_{0}}$, one need only show that $V$ satisfies the appropriate universal property.

Suppose $f \in \operatorname{Mor}_{A^{H}}\left(W_{i_{0}}, \operatorname{Res}_{H}^{G} X\right)$. If there is a $G$-map $\phi: V \rightarrow X$ such that $\left(\operatorname{Res}_{H}^{G} \phi\right) \bullet \psi=f$, then for each $g \in G$ there is a commutative diagram 


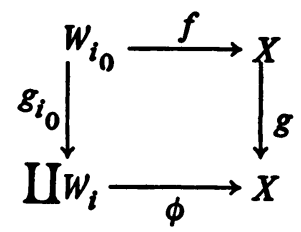

Given $i$, let $g \in G$ be such that $g_{i_{0}}: W_{i_{0}} \rightarrow W_{i} \hookrightarrow \amalg W_{i}$. Then $\phi_{i}=$ $\phi g_{i_{0}}\left(g^{-1}\right)_{i}^{i_{0}}=g f\left(g^{-1}\right)_{i}^{i_{0}}$. Thus such a $\phi$ is unique.

To show existence, define $\phi$ by $\phi_{i}=g f\left(g^{-1}\right)_{i}^{i_{0}}$, where $g \in G$ such that $g_{i_{0}}: W_{t_{0}} \rightarrow W_{i} \hookrightarrow \amalg W_{i} \cdot \phi$ is well defined: If $\tilde{g}, g: W_{t_{0}} \rightarrow W_{l} \hookrightarrow \amalg W_{l}$, then $\tilde{g}^{-1} g \in H$, so that $\tilde{g}^{-1} g f=f\left(\tilde{g}^{-1} g\right)_{i_{0}}^{i_{0}}=f\left(\widetilde{g}^{-1}\right)_{i}^{i_{0}} g_{i_{0}}^{i}$; hence $g f\left(g^{-1}\right)_{i}^{i_{0}}$ $=\tilde{g} f\left(\tilde{g}^{-1}\right)_{i}^{i_{0}}$.

LEMMA II.8. Let A have coequalizers and finite coproducts, and let $G$ be a finite group. Then

natural in arguments $A$ and $B$.

$$
(A \amalg B)_{G} \approx A_{G} \amalg B_{G},
$$

Proof. There is a natural isomorphism

$$
\operatorname{Mor}_{A}\left((A \amalg B)_{G}, X\right) \approx \operatorname{Mor}_{A} G\left(A \amalg B, F_{G}(X)\right)
$$

(Remark II.4). Since adjoints are unique, one need only show

$$
\operatorname{Mor}_{A}\left(A_{G} \amalg B_{G}, X\right) \approx \operatorname{Mor}_{A} G\left(A \amalg B, F_{G}(X)\right) \text {. }
$$

But

$$
\begin{aligned}
\operatorname{Mor}_{A}\left(A_{G} \amalg B_{G}, X\right) \approx \operatorname{Mor}_{A}\left(A_{G}, X\right) \times \operatorname{Mor}_{A}\left(B_{G}, X\right) \\
\quad \approx \operatorname{Mor}_{A} G\left(A, F_{G}(X)\right) \times \operatorname{Mor}_{A} G\left(B, F_{G}(X)\right) \approx \operatorname{Mor}_{A} G\left(A \amalg B, F_{G}(X)\right) .
\end{aligned}
$$

THEOREM II.9. Let $\boldsymbol{G}$ be a group, and let $\mathbf{A}$ have finite coproducts, coequalizers, and a "tensor product" 1 . Then if $W_{n}, W_{n}^{\prime} \in \mathrm{A}^{\mathrm{Sn}}$,

for all $T \in \mathbf{A}^{\mathbf{G}}$.

$$
\phi_{W_{n} \amalg W_{n}^{\prime}}(T)=\phi_{W_{n}}(T) \amalg \phi_{W_{n}^{\prime}}(T)
$$

Proof.

$$
\begin{aligned}
\phi_{W_{n} \amalg W_{n}^{\prime}}(T) & =\left(\left(W_{n} \amalg W_{n}^{\prime}\right) \perp T^{\perp n}\right)_{S_{n}} \\
& \approx\left(\left(W_{n} \perp T^{\perp n}\right) \amalg\left(W_{n}^{\prime} \perp T^{\perp n}\right)\right)_{S_{n}} \\
& \approx\left(W_{n} \perp T^{\perp n}\right)_{S_{n}} \amalg\left(W_{n}^{\prime} \amalg T^{\perp n}\right)_{S_{n}} \quad \text { (by Lemma II.8) } \\
& =\phi_{W_{n}}(T) \amalg \phi_{W_{n}^{\prime}}(T) . \square
\end{aligned}
$$

LEMMA II.10. Let A have finite coproducts and coequalizers, and let 
$K \subset H \subset G$ be finite groups. Let $U \in \mathbf{A}^{\mathbf{K}}$ and $W, W^{\prime} \in \mathbf{A}^{\mathbf{H}}$. Then

(i) $\operatorname{Ind}_{H}^{G}\left(W \amalg W^{\prime}\right) \approx \operatorname{Ind}_{H}^{G} W \amalg \operatorname{Ind}_{H}^{G} W^{\prime}$,

(ii) $\operatorname{Ind}_{H}^{G}\left(\operatorname{Ind}_{K}^{H} U\right) \approx \operatorname{Ind}_{K}^{G} U$,

(iii) $\left(\operatorname{Ind}_{H}^{G} W\right)_{G} \approx W_{H}$.

All the above isomorphisms are natural.

Proof. (i) The result follows from the adjointness of $\operatorname{Res}_{H}^{G}$ and $\operatorname{Ind}_{H}^{G}$ (Remark II.6) and an argument analogous to the one for Lemma II.8.

(ii) This result follows from the uniqueness of adjoints and the obvious fact that $\operatorname{Res}_{K}^{H}\left(\operatorname{Res}_{H}^{G} V\right) \approx \operatorname{Res}_{K}^{G} V$.

(iii) The proof, which is similar to the preceding one, uses the adjointness of $\operatorname{Res}_{H}^{G}$ and $\operatorname{Ind}_{H}^{G}$ and of $F_{G}$ and ()$_{G}$, and the fact that $\operatorname{Res}_{H}^{G}\left(F_{G}(X)\right) \approx$ $F_{H}(X)$.

LEMMA II.11 (FROBENIUS RECIPROCITY). Let $H \subset G$ be finite groups, and let A have finite coproducts, coequalizers, and a "tensor product" 1 . Assume there is a functor Hom: $\mathbf{A}^{\circ} \times \mathbf{A} \rightarrow \mathbf{A}$ such that

$$
\operatorname{Mor}_{A}(A \perp B, C) \approx \operatorname{Mor}_{A}(A, \operatorname{Hom}(B, C)) \text {, }
$$

natural in $A, B, C$. Then for $W \in \mathrm{A}^{\mathbf{H}}, V \in \mathrm{A}^{\mathbf{G}}$,

$$
\left(\operatorname{Ind}_{H}^{G} W\right) \perp V \approx \operatorname{Ind}_{H}^{G}\left(W \perp \operatorname{Res}_{H}^{G} V\right) \text {. }
$$

Proof. The functoriality of Hom: $\mathbf{A}^{\circ} \times \mathbf{A} \rightarrow \mathbf{A}$ induces the functor Hom: $\left(A^{G}\right)^{\circ} \times A^{G} \rightarrow A^{G}$, and clearly

$$
\operatorname{Res}_{H}^{G} \operatorname{Hom}(V, X) \approx \operatorname{Hom}\left(\operatorname{Res}_{H}^{G} V, \operatorname{Res}_{H}^{G} X\right) .
$$

The lemma now follows from the standard argument using uniqueness of adjoints.

The following theorem gives a useful simplification for some of the functors $\phi_{W_{n}}$ in the special case of the existence of an object 1 in $A$ such that $A \perp 1$ $\approx A$, natural and coherent in the sense of II, §A. The object $F_{G}(1) \in A^{G}$ will be denoted $1_{G}$, or simply 1 .

THEOREM II.12. Let $G$ be a group, let $\mathbf{A}$ have an object 1 and be as in Lemma II.11, and let Hom exist. Let $H \subset S_{n}, T \in \mathbf{A}^{\mathbf{G}}$, and $W_{n}=$ $\operatorname{Ind}_{H}^{S_{n}}$ 1. Then $\phi_{W_{n}}(T)=\left(\operatorname{Res}_{H}^{S_{n}}\left(T^{\perp n}\right)\right)_{H}$.

Proof.

$$
\begin{aligned}
\phi_{W_{n}}(T) & =\left(\left(\operatorname{Ind}_{H}^{S_{n}} 1\right) \perp T^{\perp n}\right)_{S_{n}} \\
& \approx\left(\operatorname{Ind}_{H}^{S_{n}}\left(1 \perp \operatorname{Res}_{H}^{S_{n}}\left(T^{\perp n}\right)\right)\right)_{S_{n}} \quad(\text { by Lemma II.11) } \\
& \approx\left(\operatorname{Ind}_{H}^{S_{n}}\left(\operatorname{Res}_{H}^{S_{n}}\left(T^{\perp n}\right)\right)\right)_{S_{n}} \approx\left(\operatorname{Res}_{H}^{S_{n}}\left(T^{\perp n}\right)\right)_{H} \quad(\text { by Lemma II.10). }
\end{aligned}
$$



$T^{\perp n}$.

ExAMPLES II.13. (i) If $W_{n}=\operatorname{Ind}_{1}^{S_{n}} 1$, then $\phi_{W_{n}}(T)=\left(\operatorname{Res}_{1}^{S_{n}}\left(T^{\perp n}\right)\right)_{1}=$

(ii) If $W_{n}=\operatorname{Ind}_{S_{n}}^{S_{n}} 1$, then $\phi_{W_{n}}(T)=\left(\operatorname{Res}_{S_{n}}^{S_{n}}\left(T^{\perp n}\right)\right)_{S_{n}}=\left(T^{\perp n}\right)_{S_{n}}=$ the $n$th symmetric power of $T$.

If $G$ and $H$ are groups, $A \in \mathrm{A}^{\mathrm{G}}, B \in \mathrm{A}^{\mathrm{H}}$, then the morphisms $A \stackrel{g}{\longrightarrow}$ $A, B \stackrel{h}{\longrightarrow} B$ induce the morphism $A \perp B \stackrel{g \perp h}{\longrightarrow} A \perp B$, thereby making $A \perp B$ $\in \mathrm{A}^{\mathrm{G} \times \mathrm{H}}(G \times H$ is the direct product of $G$ and $H)$. In this setting, one has the following lemma:

Lemma II.14. Let $G$ and $H$ be finite groups, and let $\mathbf{A}$ have finite coproducts, coequalizers, and a "tensor product" 1 . Assume there is a functor Hom, as in Lemma II.11. If $A \in \mathrm{A}^{\mathrm{G}}, B \in \mathrm{A}^{\mathrm{H}}$, then $(A \perp B)_{G \times H} \approx A_{G} \perp B_{H}$.

Proof. Because of the uniqueness of adjoints, one need only show

$$
\operatorname{Mor}_{\mathrm{A}} \mathrm{G \times H}\left(A \perp B, \mathrm{~F}_{G \times H}(X)\right) \approx \operatorname{Mor}_{\mathrm{A}}\left(A_{G} \perp B_{H}, X\right) \text {. }
$$

This follows from the following chain of natural isomorphisms, each easily verifiable:

$$
\begin{aligned}
& \operatorname{Mor}_{\mathrm{A}} \mathrm{G} \times \mathrm{H}(A \perp B, \quad G \times H(X)) \\
& \approx \operatorname{Mor}_{\left(\mathrm{A}^{\mathrm{H}}\right) \mathrm{G}}\left(F_{H}(A) \perp F_{G}(B), F_{G}\left(F_{H}(X)\right)\right) \\
& \approx \operatorname{Mor}_{\left(A^{H}\right)^{G}}\left(F_{H}(A), \operatorname{Hom}\left(F_{G}(B), F_{G}\left(F_{H}(X)\right)\right)\right) \\
& \approx \operatorname{Mor}_{\left(A^{H}\right)} G\left(F_{H}(A), F_{G}\left(\operatorname{Hom}\left(B, F_{H}(X)\right)\right)\right) \\
& \approx \operatorname{Mor}_{\mathrm{A} H}\left(\left(F_{H}(A)\right)_{G}, \operatorname{Hom}\left(B, F_{H}(X)\right)\right) \\
& \approx \operatorname{Mor}_{A^{H}}\left(F_{H}\left(A_{G}\right), \operatorname{Hom}\left(B, F_{H}(X)\right)\right) \\
& \approx \operatorname{Mor}_{\mathrm{A}} \mathrm{H}\left(F_{H}\left(A_{G}\right) \perp B, F_{H}(X)\right) \\
& \approx \operatorname{Mor}_{A^{H}}\left(B \perp F_{H}\left(A_{G}\right), F_{H}(X)\right) \\
& \approx \operatorname{Mor}_{\mathrm{A}}{ }^{H}\left(B \operatorname{Hom}\left({ }_{H}\left(A_{G}\right), F_{H}(X)\right)\right) \\
& \approx \operatorname{Mor}_{A^{H}}\left(B, F_{H}\left(\operatorname{Hom}\left(A_{G}, X\right)\right)\right) \approx \operatorname{Mor}_{A}\left(B_{H}, \operatorname{Hom}\left(A_{G}, X\right)\right) \\
& \approx \operatorname{Mor}_{\mathrm{A}}\left(B_{H} \perp A_{G}, X\right) \approx \operatorname{Mor}_{\mathrm{A}}\left(A_{G} \perp B_{H}, X\right) \cdot \square
\end{aligned}
$$

In the next theorem, $S_{n} \times S_{m}$ is viewed as a subgroup of $S_{n+m}$ by viewing $S_{n}$ as permuting the symbols $1,2, \cdots, n, S_{m}$ the symbols $n+1$, $n+2, \cdots, n+m$, and $S_{n+m}$ the symbols $1,2, \cdots, n+m$.

THEOREM II.15. Let $G$ be a group, let A have finite coproducts, coequal- 
izers, a "tensor product" 1 , and an object 1. Assume there is a functor Hom as in Lemma II.11. Let $W_{n} \in \mathbf{A}^{\mathbf{S}_{\mathbf{n}}}, W_{m} \in \mathbf{A}^{\mathbf{S}_{\mathbf{m}}}$, and $T \in \mathbf{A}^{\mathbf{G}}$. If $W_{n+m}=$ $\operatorname{Ind}_{S_{n} \times S_{m}}^{S_{n+m}} W_{n} \perp W_{m}$, then $\phi_{W_{n+m}}(T)=\phi_{W_{n}}(T) \perp \phi_{W_{m}}(T)$.

Proof.

$$
\begin{aligned}
& \phi_{W_{n+m}}(T)=\left(\left(\operatorname{Ind}_{S_{n} \times S_{m}}^{S_{n+m}} W_{n} \perp W_{m}\right) \perp T^{\perp n+m}\right)_{S_{n+m}} \\
& \approx\left(\operatorname{Ind}_{S_{n} \times S_{m}} S_{n+m}\left(W_{n} \perp W_{m} \perp \operatorname{Res}_{S_{n} \times S_{m}} T^{\perp n+m}\right)\right)_{S_{n+m}} \quad \text { (by Lemma II.11) } \\
& \approx\left(W_{n} \perp W_{m} \perp \operatorname{Res}_{S_{n} \times S_{m}} S_{n+m} T^{\perp n+m}\right)_{S_{n} \times S_{m}} \quad \text { (by Lemma II.10) } \\
& \approx\left(\left(W_{n} \perp T^{\perp n}\right) \perp\left(W_{m} \perp T^{\perp m}\right)\right)_{S_{n} \times S_{m}} \\
& \approx\left(W_{n} \perp T^{\perp n}\right)_{S_{n}} \perp\left(W_{m} \perp T^{\perp m}\right)_{S_{m}} \quad \text { (by Lemma II.14) } \\
& \quad=\phi_{W_{n}}(T) \perp \phi_{W_{m}}(T) . \square
\end{aligned}
$$

C. The main theorem and corollary. Let $G$ be a group and $\mathbf{A}$ have finite coproducts, a "tensor product" 1 , and an object 1 . Define the Grothendieck ring $K_{0}\left(\mathrm{~A}^{\mathrm{G}}\right)$ to consist of all finite formal sums $\Sigma_{i} n_{i}\left[T_{i}\right] \quad\left(n_{i} \in Z\right)$ of $G$-objects $T_{i}$ in $A$, modulo the relations

(i) $\left[T_{1}\right]=\left[T_{2}\right]$ if $T_{1} \cong T_{2}$ as $G$-objects,

(ii) $\left[T_{1} \amalg T_{2}\right]=\left[T_{1}\right]+\left[T_{2}\right]$.

Clearly, $K_{0}\left(\mathrm{~A}^{\mathrm{G}}\right)$ is an abelian group; the "tensor product" 1 , together with the object $1 \in A^{G}$, gives $K_{0}\left(A^{G}\right)$ the structure of a commutative ring with identity, i.e. $\left[T_{1}\right]\left[T_{2}\right]=\left[T_{1} \perp T_{2}\right]$. When the meaning is clear, brackets will be omitted, e.g., $\left[T_{1}\right]-\left[T_{2}\right]$ will appear as $T_{1}-T_{2}$.

EXAMPLES II.16. (i) Let $G$ be a finite group and $A$ the category of finite sets. Then $A^{G}=G$-sets. Let $\perp$ be the cartesian product, and 1 be any one-element $G$-set. Then $K_{0}\left(A^{G}\right)$ is the Burnside ring of $G, B(G)$. (See I, §B.)

(ii) Let $G$ be a finite group and $A$ the category of finite-dimensional vector spaces over $\mathbf{C}$. Then $\mathbf{A}^{\mathbf{G}}=\mathbf{G}$-modules. Let $\perp$ be the tensor product $\otimes$, and 1 be the one-dimensional $G$-module with trivial $G$-action. Then $K_{0}\left(\mathrm{~A}^{\mathrm{G}}\right)$ is the representation ring of $G, R(G)$ (see $\mathrm{I}, \S \mathrm{C}$ ).

REMARK II.17. In the above examples, $\left[T_{1}\right]=\left[T_{2}\right]$ implies $T_{1} \cong T_{2}$ as $G$-objects (see I, $\S \mathrm{B}, \S \mathrm{C}$ ). This is not the case in general; in particular, if $A$ is the category of vector bundles over a space $X$, then $[E]=[F]$ implies only that $E \oplus \mathrm{n} \cong F \oplus \mathrm{n}$, where $\mathrm{n}$ is the trivial bundle of dimension $n$ [3, Appendix]. 
Let $H \subset G$ be finite groups. Let $\mathrm{A}$ have finite coproducts, coequalizers, a "tensor product" $\perp$, and an object 1. $P\left(A^{G}\right)$ is defined to be the subring of $K_{0}\left(\mathrm{~A}^{\mathrm{G}}\right)$ generated by $\left\{\operatorname{Ind}_{H}^{G} 1 \mid H\right.$ a subgroup of $\left.G\right\}$.

Proposition II.18. Let $H \subset G$ be finite groups. If $W=1_{H}$, then $\operatorname{Ind}_{H}^{G} W=\amalg_{\bar{x} \in G / H} 1_{\bar{x}}$ with G-action given by

$$
g: 1_{\bar{x}} \stackrel{1_{1}}{\longrightarrow} 1_{\overline{g x}} \smile \amalg_{1_{\bar{x}}}
$$

Proof. Let $\psi: 1_{\overline{1}} \hookrightarrow \amalg 1_{\bar{x}}$. One need only show that $\left(\amalg 1_{\bar{x}}, \psi\right)$ satisfies the appropriate universal property. Clearly, $\psi: 1_{\overline{1}} \rightarrow \operatorname{Res}_{H}^{G}\left(\mathbf{H 1}_{\bar{x}}\right)$ is an $H$-map. If $f \in \operatorname{Mor}{ }_{\mathrm{A}^{H}}\left(1_{H}, \operatorname{Res}_{H}^{G} X\right)$, there is a unique $\phi \in \operatorname{Mor}_{\mathrm{A}} \mathrm{G}\left(111_{\bar{x}}, X\right)$ such that $\left(\operatorname{Res}_{H}^{G} \phi\right){ }^{\circ} \psi=f$, namely $\phi_{\bar{x}} x f 11_{\bar{x}}^{\overline{1}}$ for all $\bar{x} \in G / H$.

Proposition II.19. Every element in $P\left(\mathrm{~A}^{G}\right)$ is of the form $\Sigma_{i} n_{i} \operatorname{Ind}_{H_{i}}^{G}$, where $n_{i} \in \mathrm{Z}$ and $H_{i}$ is a subgroup of $G$.

Proof.

$$
\begin{aligned}
& \left(\operatorname{Ind}_{H}^{G} 1\right) \perp\left(\operatorname{Ind}_{K}^{G} 1\right) \approx\left(\coprod_{\bar{x} \in G / H} 1_{\bar{x}}\right) \perp\left(\coprod_{\bar{y} \in G / K} 1_{\bar{y}}\right) \quad \text { (by Proposition II.18) } \\
& \approx \coprod_{\bar{x}, \bar{y}}\left(1_{\bar{x}} \perp 1_{\bar{y}}\right) \\
& \approx \coprod_{\bar{x}, \bar{y}} 1_{(\bar{x}, \bar{y})} \approx \prod_{\substack{G \text {-orbits } \alpha \\
\text { of } G / H \times G / K}}\left(\coprod_{(\bar{x}, \bar{y}) \in \alpha} 1_{(\bar{x}, \bar{y})}\right) \\
& \approx \coprod_{G \text {-orbits } \alpha} \operatorname{Ind}_{H_{\alpha}}^{G} 1 \text { (by Proposition II.7). } \square
\end{aligned}
$$

The canonical $S_{k}$-object in A, denoted $X_{k}$, is defined to be $\operatorname{Ind}_{S_{1} \times s_{k-1}} s_{k}$. REMARK II.20. From Proposition II.18, it follows that

$$
X_{k}=\prod_{\bar{\sigma} \in S_{k} /\left(S_{1} \times S_{k-1}\right)} \mathbf{1}_{\bar{\sigma}} \text {. }
$$

Since $\bar{\sigma}=\bar{\tau}$ in $S_{k} /\left(S_{1} \times S_{k-1}\right)$ iff $\tau(1)=\sigma(1)$, each $S_{1} \times S_{k-1}$-orbit of $S_{k}$ consists of precisely those $\sigma \in S_{k}$ which send 1 to the same symbol $j$. Hence $X_{k}=\amalg_{j=1}^{k} 1_{j}$, where $\sigma \in S_{k}$ acts by

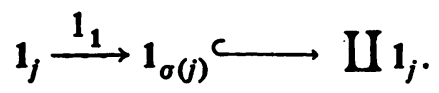

For examples, see I.1(iii) and I.6(ii).

Let $G$ be a group, and $\mathbf{A}$ have finite coproducts, coequalizers, a "tensor product" 1 , and object 1 . Let $\phi_{0}: \mathbf{A}^{\mathbf{G}} \rightarrow \mathbf{A}^{\mathbf{G}}$ be the functor sending $A \in$ 
$A^{G}$ to $1_{G} \cdot \phi_{0}$ and the functors $\phi_{W_{n}}$ arising from all positive integers $n$ and all $W_{n} \in \mathrm{A}^{\mathbf{S}_{\mathbf{n}}}$ (see II, $\S \mathrm{A}$ ) will be called $S$-operations. If $\mathbf{A}=G$-modules (see $\mathrm{I}, \S \mathrm{C}$ ), the $S$-operations generate what are known as $\lambda$-operations. If $A=$ $G$-sets (see I, §B), the $S$-operations will be referred to as $\beta$-operations.

For $T \in A^{G}$, let $\langle T\rangle$ denote the subring of $K_{0}\left(A^{G}\right)$ generated by $\{[\phi(T)] \mid \phi$ an $S$-operation\}. If there is a functor Hom as in Lemma II.11, then Theorem II.15 says that every element in $\langle T\rangle$ is a finite sum $\Sigma_{\alpha} q_{\alpha}\left[\phi_{\alpha}(T)\right]$, where $q_{\alpha} \in Z, \phi_{\alpha}$ an $S$-operation.

Summarizing, the ring $K_{0}\left(A^{G}\right)$ and subrings $P\left(A^{G}\right)$ and $\langle T\rangle$ have been constructed. The main theorem and its immediate corollary apply when $G=S_{k}$ and $T=X_{k}$ :

MAIN THEOREM II.21. Let A have finite coproducts, coequalizers, a "tensor product" 1 , and an object 1. Assume there is a functor Hom as in Lemma II.11. Then for each positive integer $k, P\left(A^{S_{k}}\right) \subset\left\langle X_{k}\right\rangle$.

Corollary II.22. Same hypothesis as above. Suppose $P\left(A^{S_{k}}\right)=K_{0}\left(A^{S_{k}}\right)$. Then $\left\langle X_{k}\right\rangle=K_{0}\left(\mathrm{~A}^{\mathrm{S}_{\mathrm{k}}}\right)$.

LEMma II.23. Same hypothesis as above. Let $H \subset S_{n}$ and $W_{n}=$ $\operatorname{Ind}_{H}^{S_{n}}$ 1. Then

$$
\phi_{W_{n}}\left(X_{k}\right)=\coprod_{\gamma} \operatorname{Ind}_{H_{\gamma}}^{s_{k}} 1,
$$

for some collection of subgroups $H_{\gamma}$ of $S_{k}$. Here, $\gamma_{1} \neq \gamma_{2}$ need not imply $H_{\gamma_{1}} \neq H_{\gamma_{2}}$.

This lemma does not imply $\left\langle X_{k}\right\rangle \subset P\left(A^{S_{k}}\right)$, since $\left\langle X_{k}\right\rangle$ is obtained from all $S$-operations $\phi_{W_{n}}$, and if $P\left(\mathrm{~A}^{\mathrm{S}_{\mathrm{n}}}\right) \neq K_{0}\left(\mathrm{~A}^{\mathrm{s}_{\mathrm{n}}}\right), W_{n}$ need not be a linear combination of objects $\operatorname{Ind}_{H} S_{n} 1$.

Proof OF LEMMA. By Theorem II.12, $\phi_{W_{n}}\left(X_{k}\right)=\left(\operatorname{Res}_{H}^{S_{n}}\left(X_{k}^{\perp n}\right)\right)_{H}$. Since

$$
\begin{aligned}
X_{K}^{\perp n}=\left(\coprod_{j=1}^{k} 1_{j}\right)^{\perp n} & \approx \coprod_{\substack{\left.j_{1}, \cdots, j_{n}\right) \\
1<j_{i}<k}}\left(1_{j_{1}} \perp \cdots \perp 1_{j_{n}}\right) \\
& \approx \prod_{\substack{\left.j_{1}, \cdots, j_{n}\right) \\
1<j_{i}<k}} 1_{\left(j_{1}, \cdots, j_{n}\right)},
\end{aligned}
$$

we have

$$
\phi_{W_{n}}\left(X_{k}\right)=\left(\operatorname{Res}_{H}^{s_{n}}\left(\coprod_{1<j_{i}<k} 1\left({V_{1}}_{1}, \cdots, j_{n}\right)\right)\right)_{H}
$$


$\sigma \in S_{n}$ acts on $\amalg 1_{\left(j_{1}, \cdots, J_{n}\right)}$ by

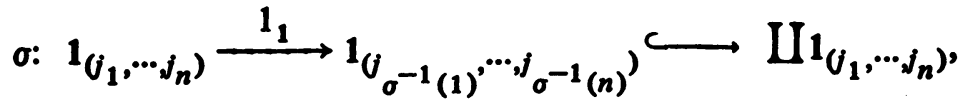

and $-g \in S_{k}$ by

$$
g: 1_{\left(j_{1}, \cdots, j_{n}\right)} \stackrel{1_{1}}{\longrightarrow} 1_{\left(g\left(j_{1}\right), \cdots, g\left(j_{n}\right)\right)} \hookrightarrow \amalg 1_{\left(j_{1}, \cdots, j_{n}\right)} ;
$$

moreover, $\sigma g=g \sigma: \amalg 1_{\left(j_{1}, \cdots, j_{n}\right)} \rightarrow \amalg 1_{\left(j_{1}, \cdots, j_{n}\right)}$.

Let $J=\left\{\left(j_{1}, \cdots, j_{n}\right) \mid 1 \leqslant j_{i} \leqslant k\right\}$. By the above, $H \subset S_{n}$ acts on the set $J$. For $j \in J$, let $\bar{j}$ denote the orbit $H j$. Using equation (1), it is not hard to show that $\phi_{W_{n}}\left(X_{k}\right)=\amalg_{H \text {-orbits } j^{-1}}$, where $g \in S_{k}$ acts by $g: 1_{j} \stackrel{1_{1}}{\longrightarrow}$ $\mathbf{1}_{\bar{g} \boldsymbol{T}} \hookrightarrow \mathrm{I1} \mathbf{1}_{\Gamma}$. Let

$$
\pi: \coprod_{j} 1_{j} \rightarrow \coprod_{\Gamma} 1_{\Gamma}
$$

be the map induced from $1_{j} \stackrel{1_{1}}{\longrightarrow} 1_{\Gamma} \hookrightarrow \amalg_{\digamma} 1_{\digamma}$. It is straightforward to show that $\left(\amalg_{\digamma} \mathbf{1}_{\Gamma}, \pi\right)$ satisfies the universal property defining $\left(\amalg_{j \in J} \mathbf{1}_{j}\right)_{H}$. Hence as an object in $A, \phi_{W_{n}}\left(X_{k}\right)=\amalg_{\Gamma^{1}}{ }$. Since, for $g \in S_{k},(\pi g) h=\pi h g=\pi g: \amalg 1_{j} \rightarrow$

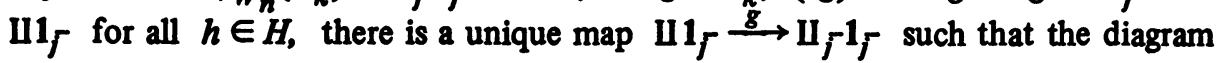

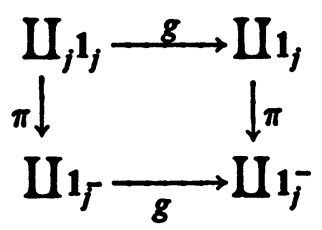

commutes, and hence is determined by the commutative diagram:

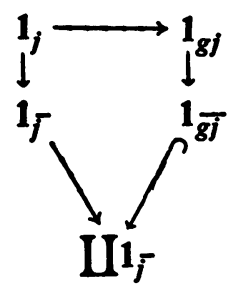

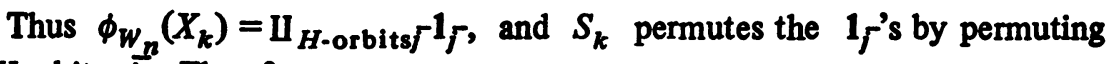
the $H$-orbits $\vec{j}$. Therefore,

$$
\phi_{W_{n}}\left(X_{k}\right) \approx \coprod_{\Gamma} 1_{\Gamma} \approx \prod_{s_{k} \text {-orbits } \gamma}\left(\coprod_{\Gamma \in \gamma} 1_{\Gamma}\right) \approx \operatorname{Ind}_{H_{\gamma}}^{s_{k}} 1_{\Gamma_{0}}
$$

(by Proposition II.7), where $\mathbf{1}_{\digamma_{0}}$ is one of the $1_{\digamma}$ 's and $H_{\gamma}$ is its isotropy group. $\square$

Let $H \subset S_{k}$ and $m$ be a nonnegative integer. $H$ is said to be divisible 
by $S_{m}$ if $H$ is conjugate to $M \times S_{m}$ (as subgroups of $S_{k}$ ) for some subgroup $M$ of $S_{k-m}$. Here, " $H$ conjugate to $M \times S_{0}$ " means $H$ is conjugate to some subgroup $M$ of $S_{k}$; " $H$ conjugate to $M \times S_{k}$ " means $H$ is conjugate to $S_{k}$. Clearly $H$ is always divisible by $S_{0}$.

Proof of main theorem. It is enough to show that if $H \subset S_{k}$ is divisible by $S_{m}$ for some $m, 0 \leqslant m \leqslant k$, then $\operatorname{Ind}_{H}^{S_{k}} 1 \in\left\langle X_{k}\right\rangle$. The proof is by induction (backwards) on $m$ :

(i) If $m=k$, then $H=S_{k}$ and $\operatorname{Ind}_{H}^{S_{k}} 1=1_{S_{k}}=\phi_{0}\left(X_{k}\right) \in\left\langle X_{k}\right\rangle$.

(ii) Suppose $m<k$ and assume that if $m<m^{\prime} \leqslant k$, then $H$ divisible by $S_{m^{\prime}} \Rightarrow \operatorname{Ind}_{H}^{S_{k}} 1 \in\left\langle X_{k}\right\rangle$. Let $H$ be divisible by $S_{m}$. Then $\operatorname{Ind}_{H}^{s_{k}} 1=$

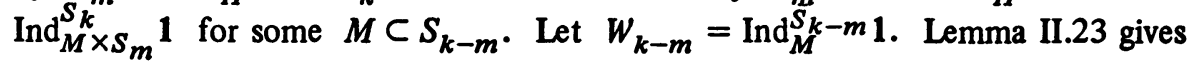

$$
\phi_{W_{k-m}}\left(X_{k}\right)=\coprod_{s_{k} \text {-orbits } \gamma}\left(\coprod_{j \in \gamma} 1_{j}\right)=\coprod_{\gamma} \operatorname{Ind}_{H_{\gamma}}^{s_{k}} 1 .
$$

Recall that $J=\left\{\left(j, \cdots, j_{k-m}\right) \mid 1 \leqslant j_{i} \leqslant k\right\}$ is an $S_{k}$-set and an $M$-set, that $S_{k}$ permutes the $M$-orbits $\bar{j}$ of $J$, and that $\gamma$ runs through the $S_{k}$-orbits of the set of $M$-orbits of $J$.

Direct computation shows that the $M$-orbit $(\overline{1, \cdots, k-m})$, which is in some $S_{k}$-orbit $\gamma_{0}$, has isotropy group $H_{\gamma_{0}}=M \times S_{m}$. Moreover, $\left(\overline{j_{1}, \cdots, j_{k-m}}\right) \in \gamma_{0}$ whenever all the $j_{i}$ 's are distinct, since $S_{k}$ is $(k-m)$ fold transitive. Thus if $\left(\overline{j_{1}, \cdots, j_{k-m}}\right) \in \gamma \neq \gamma_{0}, j_{i}=j_{t}$ for some $i \neq t$; hence its isotropy group $H_{\gamma}$ is of the form $K \times S_{m^{\prime}}$, for some $m^{\prime}>m$ and $K \subset$ $S_{k-m}$. Since $H_{\gamma}$ is divisible by $S_{m^{\prime}}$, for some $m^{\prime}>m$ if $\gamma \neq \gamma_{0}, \operatorname{Ind}_{H_{\gamma}} S_{k} \in$ $\left\langle X_{k}\right\rangle$ for all $\gamma \neq \gamma_{0}$ by induction hypothesis. Thus

$$
\operatorname{Ind}_{H}^{s_{k}} 1=\operatorname{Ind}_{M \times s_{m}}^{s_{k}} 1=\phi_{W_{k-m}}\left(X_{k}\right)-\sum_{\gamma \neq \gamma_{0}} \operatorname{Ind}_{H}^{S_{k}} 1 \in\left\langle X_{k}\right\rangle
$$

The proof is completed by induction. $\square$

\section{Applications and Open Questions}

A. Unigeneration of the $\lambda$-ring $R\left(S_{k}\right)$. It is well known that $R\left(S_{k}\right)$ is a free Z-module with basis $\left\{\operatorname{Ind}_{S_{k_{1}} \times S_{k_{2}} \times \cdots s_{k_{s}}} 1 \mid k_{i} \geqslant 1, \Sigma_{i} k_{i}=k\right.$ \} [5, Chapter III]. Therefore, $P\left(\mathrm{~A}^{\mathrm{S}_{\mathbf{k}}}\right)=K_{0}\left(\mathrm{~A}^{\mathrm{S}_{\mathrm{k}}}\right)=R\left(S_{k}\right)$, where $\mathrm{A}=$ finite-dimensional vector spaces over C. Corollary II.22 now implies $R\left(S_{k}\right)=\left\langle X_{k}\right\rangle$, and Theorem II.15 gives that every element of $R\left(S_{k}\right)$ is a linear combination of $\left\{\left[\phi\left(X_{k}\right)\right] \mid \phi\right.$ an $S$-operation\}.

Moreover, every $S$-operation is a linear combination of symmetric power operations:

$$
W_{n} \in R\left(S_{n}\right) \Rightarrow\left[W_{n}\right]=\left[W_{n}^{\prime}\right]-\left[W_{n}^{\prime \prime}\right],
$$


where

$$
W_{n}^{\prime}=\sum \alpha_{\mu} \operatorname{Ind}_{S_{\mu_{1}} \times \cdots \times S_{\mu_{s}}}^{S_{n}} 1, \quad W_{n}^{\prime \prime}=\sum \beta_{\nu} \operatorname{Ind}_{S_{\nu_{1}} \times \cdots \times s_{\nu_{t}}} S_{n}
$$

with $\alpha_{\mu}, \beta_{\nu}$ positive integers.

$$
\begin{aligned}
{\left[W_{n}^{\prime}\right] } & =\left[W_{n}\right]+\left[W_{n}^{\prime \prime}\right]=\left[W_{n} \amalg W_{n}^{\prime}\right] \Rightarrow W_{n}^{\prime} \cong W_{n} \amalg W_{n}^{\prime \prime} \\
& \Rightarrow \phi_{W_{n}^{\prime}}(T)=\phi_{W_{n}}(T) \amalg \phi_{W_{n}^{\prime \prime}}(T) \text { (by Theorem II.9) } \\
& \Rightarrow\left[\phi_{W_{n}}(T)\right]=\left[\phi_{W_{n}^{\prime}}(T)\right] \amalg\left[\phi_{W_{n}^{\prime \prime}}(T)\right] .
\end{aligned}
$$

Since

$$
\operatorname{Ind} S_{n_{1} \times S_{n_{2}}}^{S_{n}} 1=\operatorname{Ind}_{S_{n_{1}}^{n} \times S_{n_{2}}}(1 \otimes 1)
$$

etc., Theorem II.15 implies $\phi_{W_{n}}(T)$ is a linear combination of $\left\{h_{n_{1}}(T) \otimes\right.$ $\left.h_{n_{2}}(T) \otimes \cdots \otimes h_{n_{s}}(T) \mid n_{i} \geqslant 0\right\}$, where $h_{0}=\phi_{0}$, and $h_{n}=\phi_{W_{n}}$ for $n>0$ and $W_{n}=\operatorname{Ind}_{S_{n}} S_{n}$. The $h_{i}$ 's are, of course, symmetric power operations (see Example II.13(ii)).

Combining the two paragraphs above, one obtains the result that every element of $R\left(S_{k}\right)$ is a linear combination of $\left\{h_{n_{1}}\left(X_{k}\right) \otimes \cdots \otimes h_{n_{s}}\left(X_{k}\right) \mid n_{i} \geqslant 0\right\}$. Thus $R\left(S_{k}\right)$ is generated by the single element $X_{k}$ if symmetric powers are included with the standard ring operations. Since $\lambda$-operations generate symmetric power operations [2], [5], $X_{k}$ generates $R\left(S_{k}\right)$ as a $\lambda$-ring.

REMARK III.1. Although $R\left(S_{k}\right)$ is unigenerated as a $\lambda$-ring, it is not unigenerated as a ring, i.e., $R\left(S_{k}\right) \neq \mathrm{Z}[T]$ for all $T \in R\left(S_{k}\right)$. The first counterexample is $R\left(S_{4}\right)$ :

If $R\left(S_{4}\right)=\mathrm{Z}[T]$, then the ring $\mathrm{Z} / 2 \otimes_{\mathrm{Z}} R\left(S_{4}\right)$ is unigenerated as a $\mathrm{Z} / 2$ module. Since $R\left(S_{4}\right)$ is a free Z-module of rank 5 (see Proposition I.9), Z/2 $\otimes_{\mathrm{Z}} R\left(S_{4}\right)$ is a free $\mathrm{Z} / 2$-module of rank 5 . By writing out its multiplication table $\left(\mathrm{Z} / 2 \otimes_{\mathrm{Z}} R\left(S_{4}\right)\right.$ has only $2^{5}$ elements), one can show that no element generates all of $\mathrm{Z} / 2 \otimes_{\mathrm{Z}} R\left(S_{4}\right)$.

B. A unigeneration theorem for $B\left(S_{k}\right), B(G)$ is a free Z-module with basis $\left\{G / H_{\alpha}\right\}$, where $\left\{H_{\alpha}\right\}=$ a set of representatives of the conjugacy classes of subgroups of $G$ (Proposition I.5). Clearly, if $A=$ finite sets, then $\operatorname{Ind}_{H}^{G} 1=$ the $G$-set $G / H$ (see Proposition II.18). Thus $P\left(A^{G}\right)=K_{0}\left(A^{G}\right)=B(G)$. Hence Corollary II.22 implies that $B\left(S_{k}\right)=\left\langle X_{k}\right\rangle$. Thus $S$-operations (here called "ß-operations") applied to $X_{k}$ generate all of $B\left(S_{k}\right)$.

REMARK III.2. $B\left(S_{k}\right)$ is not, in general, generated by one element as a ring, since the ring homomorphism $B(G) \rightarrow R(G)$ defined by $T \mapsto$ vector 
space with basis $\left\{v_{t}\right\}_{t \in T}$ (see Examples I.6(ii), (ii')) is onto if $G=S_{k}$ [5, Chapter III], and therefore the ring $B\left(S_{4}\right)$ is not unigenerated since $R\left(S_{4}\right)$ is not (see Remark III.1).

REMARK III.3. Applying sums and products of symmetric power operations $h_{n}$ to $X_{k}$ does not, in general, give all of $B\left(S_{k}\right) . B\left(S_{3}\right)$ is a counterexample:

The nonconjugate subgroups of $S_{3}$ are $1, S_{1} \times S_{2}, S_{3}$ and $A_{3}$ (= the even permutations in $\left.S_{3}\right)$. Proposition I.5 now says that $B\left(S_{3}\right)$ is a free Zmodule with basis $S_{3} / 1, S_{3} / S_{1} \times S_{2}, S_{3} / S_{3}, S_{3} / A_{3}$. Note that $S_{3} / S_{1} \times S_{2}=$ $X_{3}$ (see Proposition II.19) and $S_{3} / S_{3}=1 . B\left(S_{3}\right)$ is now completely described by the following multiplication table, which is obtained easily by direct calculation using Propositions I.2, I.3, and I.4:

\begin{tabular}{c|c|c|c|c|}
\multicolumn{1}{c}{1} & \multicolumn{1}{c}{$S_{3} / A_{3}$} & $X_{3}$ & $S_{3} / 1$ \\
\cline { 2 - 5 }$S_{3} / A_{3}$ & 1 & $S_{3} / A_{3}$ & $X_{3}$ & $S_{3} / 1$ \\
\hline$X_{3}$ & $S_{3} / A_{3}$ & $2 S_{3} / A_{3}$ & $S_{3} / 1$ & $2 S_{3} / 1$ \\
\hline$S_{3} / 1$ & $X_{3}$ & $S_{3} / 1$ & $S_{3} / 1+X_{3}$ & $3 S_{3} / 1$ \\
\hline$S_{3} / 1$ & $2 S_{3} / 1$ & $3 S_{3} / 1$ & $6 S_{3} / 1$ \\
\hline
\end{tabular}

Now suppose that the symmetric power operations $h_{n}$ applied to $X_{3}$ give all of $B\left(S_{3}\right)$. Then, in particular, $S_{3} / A_{3}$ could be expressed as a finite sum

$$
\sum a_{i_{1} \ldots s} h_{n_{i_{1}}}\left(X_{3}\right) h_{n_{i_{2}}}\left(X_{3}\right) \cdots h_{n_{i_{s}}}\left(X_{3}\right) \text {, where } a_{i_{1} \ldots s} \in \mathbf{Z} \text {. }
$$

From the multiplication table, it is clear that one of the $h_{n}\left(X_{3}\right)$ 's above must be of the form $n_{1} 1+n_{2} S_{3} / A_{3}+n_{3} X_{3}+n_{4} S_{3} / 1$, with $n_{2} \neq 0$. But for all $n \geqslant$ $0, h_{n}\left(X_{3}\right)=n_{1} 1+n_{3} X_{3}+n_{4} S_{3} / 1$ for some $n_{i} \in Z$ : An element $\left(x_{1}, x_{2}, \cdots\right.$, $\left.x_{n}\right)$ in an $S_{n}$-orbit of $X_{3}^{n}$ is made up of $\mu_{1} 1$ 's, $\mu_{2} 2$ 's, $\mu_{3}$ 3's, where $\mu_{1}+$ $\mu_{2}+\mu_{3}=n$, and the 3-tuple $\left(\mu_{1}, \mu_{2}, \mu_{3}\right)$ uniquely determines the $S_{n}$-orbit. If the $\mu_{i}$ 's are all different, then the $S_{3}$-orbit of $\left(X_{3}^{n}\right)_{S_{n}}$ which contains the $S_{n}$-orbit corresponding to $\left(\mu_{1}, \mu_{2}, \mu_{3}\right)$ is $S_{3} / 1$. If exactly two of the $\mu_{i}$ 's are the same, then the $S_{3}$-orbit is $S_{3} / S_{1} \times S_{2}$. If $\mu_{1}=\mu_{2}=\mu_{3}$, then the $S_{3}$ orbit is $S_{3} / S_{3}$. Therefore, $S_{3} / A_{3}$ never arises.

Thus $B\left(S_{k}\right)$ is generated by $X_{k}$ if all the $\beta$-operations are used, but is, in general, not generated by $X_{k}$ if only symmetric power operations are used. Hence $\beta$-operations include, but are not the same as, symmetric powers. 
C. Some open questions. Since the $S$-operations in the linear representation theory case are generated by symmetric power operations (see III, §A), which are defined on all of $R(G)$ (see I, $\S \mathrm{C}$ ), $S$-operations extend to operations on $R(G)$, thus making $R(G)$ a " $\lambda$-ring". The unigeneration of $R\left(S_{k}\right)$ can be phrased:

There is an onto " $\lambda$-ring homomorphism"

$$
\Lambda \rightarrow R\left(S_{k}\right), \quad a_{1} \mapsto X_{k},
$$

where $\Lambda$ is the "free $\lambda$-ring on one generator" $a_{1} \in \Lambda$. Hence $R\left(S_{k}\right) \cong \Lambda / I$, for some $\lambda$-ideal $I$. A reasonable description of this $\lambda$-ideal, in particular, a canonical set of generators, is unknown.

In the case of permutation representations, i.e., $G$-sets, the corresponding theory of " $\beta$-ring" which would allow extending the $\beta$-operations to all of $B(G)$ is not known.

\section{REFERENCES}

1. M. Atiyah, Power operations in K-theory, Quart. J. Math. Oxford Ser. (2) 17 (1966), 165-193. MR 34 \#2004.

2. M. Atiyah and D. O. Tall, Group representations, $\lambda$-rings, and the J-homomorphism, Topology 8 (1969), 253-297. MR 39 \#5702.

3. H. Bass, Algebraic K-theory, Benjamin, New York 1968. MR 40 \#2736.

4. A. Dress, Representations of finite groups. 1. The Burnside ring (mimeographed notes, Bielefeld, 1971).

5. D. Knutson, $\lambda$-rings and the representation theory of the symmetric group, Lecture Notes in Math., vol. 308, Springer-Verlag, Berlin and New York, 1973.

6. B. Mitchell, Theory of categories, Academic Press, New York, 1965. MR 34 \#2647.

7. N. Saavedra Rivano, Categories Tannakiennes, Lecture Notes in Math., vol. 265, Springer-Verlag, Berlin and New York, 1972.

8. J.-P. Serre, Representations linéaires des groupes finis, Hermann, Paris, 1967. MR 38 \#1190.

DEPARTMENT OF MATHEMATICS, UNIVERSITY OF MICHIGAN, ANN ARBOR, MICHIGAN 48104 